\title{
Alfuzosin in the Treatment of Chronic Prostatitis/Chronic Pelvic Pain Syndrome
}

Nickel JC, Krieger JN, McNaughton-Collins M, et al.: Alfuzosin and symptoms of chronic prostatitis-chronic pelvic pain syndrome. N Engl J Med 2008, 359:2663-2673.

Rating: • Of importance.

Introduction: $\alpha$-Blockers are commonly used to treat symptoms of chronic prostatitis/chronic pelvic pain syndrome (CP/CPPS). Results from clinical trials on the efficacy of $\alpha$-blockers for this condition are mixed. The authors of this study studied alfuzosin in a large group of men who were both $\alpha$-blocker naïve and had a diagnosis of CP/CPPS for less than 2 years.

Aims: To determine the efficacy of alfuzosin for treating symptoms of CP/CPPS.

Methods: A randomized, placebo-controlled trial was conducted at 11 tertiary care centers in North America and one in Malaysia. Patients who participated were diagnosed with CP/ CPPS within 2 years of the trial and had not previously been on $\alpha$-adrenergic antagonists. Men were randomly assigned to alfuzosin, $10 \mathrm{mg} / \mathrm{d}$, or a matching placebo for 12 weeks in a 1:1 ratio. The primary outcome measure was a four-point decrease from baseline at 12 weeks on the National Institutes of Health Chronic Prostatitis Symptom Index (NIH-CPSI). Secondary end points included a global response assessment, McGill Pain Questionnaire, Medical Outcomes Study Short Form Health Survey 12 for quality of life, Hospital Anxiety and Depression Scale, International Index of Erectile Function, and the Male Sexual Health Questionnaire. Major exclusion criteria included previous treatment with $\alpha$-blockers for CP/CPPS, urinary tract infection, current sexually transmitted disease, or genitourinary cancer.

Results: A total of 272 men were randomly assigned. A decrease of at least four points on the NIH-CPSI was seen in $49.3 \%$ of patients in both study groups. The only significant difference observed between groups on secondary end points was improvement from baseline to 12 weeks on the ejaculatory function on the Male Sexual Health Questionnaire in men receiving alfuzosin compared with those receiving placebo.
Discussion: Several smaller studies showed significant benefit of $\alpha$-blockers in men with CPPS. However, this study showed essentially no difference. This was consistent with another large, randomized, multicenter trial that enrolled patients who were not necessarily naïve to $\alpha$-blockers.

\section{Comments}

One of the key points of this study was that the end point was the NIH-CPSI, with the score driven largely by the pain questions. Thus, one can conclude that $\alpha$ blockers may not be effective for treatment of the pain associated with CP/CPPS. Only two questions in the NIH-CPSI symptom score relate to urinary function. This study should not be taken to mean that one should never use $\alpha$-blockers to treat men with CP/CPPS; those with voiding symptoms may benefit from treatment with these medications. Another interesting point raised in the discussion is that it is not known whether men who are truly newly diagnosed (ie, symptoms of short duration) would benefit.

\section{Acknowledgment}

Dr. Michel Pontari is affiliated with the Department of Urology at the Temple University School of Medicine, where he is a Professor of Urology. Correspondence should be sent to 3401 North Broad Street, Zone C, Suite 330, Philadelphia, PA 19140, USA. E-mail: pontarm@ tuhs.temple.edu.

\section{Disclosure}

Dr. Pontari has served as a consultant for Sanofi-Aventis and as a clinical investigator for Pfizer. 